remains dry. In the subsequent two or three days lotions introduced through the catheter for bladder lavage emerge suprapubically, but even this soon ceases.

\section{Operative Results}

Since I adopted this technique, more than two years ago, my personal results have greatly improved. It is true to say that from no private patient's wound have I removed any slough, however small. The whole wound, with the exception of the tube track, has in every case healed per primam, and complete wound dryness has never been delayed beyond a fortnight. The patients leave their beds, as a rule, in about three weeks. Pyuria gives little or no trouble, and there is no difficulty in maintaining an acid and healthy urine. The deposition of phosphates has never been so much as suggested. It is scarcely necessary to add that the personal wellbeing of the patients is for the most part excellent. Claims but little inferior may also be made for my hospital cases. The methods are quite superior to any I have previously tried, or seen tried, and will, I venture to hope, be given a trial by other members of the profession.

REFERENCES

1 Arch. f. Verdauungskrankh., 1899.

2 Virchow's Arch., 1890

3 Compt. rend. Acad. des. Sci., 1886.

\section{CYSTIC DILATATION OF THE COMMON BILE DUCT}

Report of a Case with Unusual features

BY

JAMES H. SAINT, M.D., M.S., F.R.C.S.

SURGICAL REGISTRAR, ROYAL VICTORIA INFIRMARY, NEWCASTLEUPON-TYNE; IATE FELLOW IN SURGERY, MAYO CLINIC, ROCHESTER, MINNESOTA, U.S.A.

(With Special Plate)

The comparative rarity of cystic dilatation of the common bile duct, usually referred to as common duct cyst, may be judged from the number of recorded cases. McConnell ${ }^{1}$ was able to collect records of only thirty-five cases up to the end of 1917. Ten years later the number was reported by Judd and Greene ${ }^{2}$ to have increased to sixty-four. They state that in 17,381 operations performed on the biliary tract at the Mayo Clinic a common duct cyst was encountered only once. The increasing interest in the condition due to the more frequent writings on the subject during the past few years has resulted in the number being increased to ninety-three in 1930, as reported by Wilson. ${ }^{3}$

It is only within recent years that it has been diagnosed before operation, the first person to do this being Neugebauer ${ }^{4}$ in 1924. Since then only Taylor ${ }^{5}$ and Iselin have attained this rare distinction. The case reported below was no exception to the general rule of failure of pre-operative diagnosis, for its clinical features were so unusual that the thought of the possibility of a common duct cyst being present was never even entertained.

Case History and Examination

J. C., a married woman, aged 43 years, was admitted to the Royal Victoria Infirmary on September 9th, 1931, with the complaint of severe upper abdominal pain. The onset of this pain occurred some six hours before admission. For the next two hours she was able to go about, but the pain then became worse and she went to bed. Two hours later she vomited a small quantity of greenish, bitter material; this gave her a little relief for a while, and then the pain began to increase in severity, and continued to do so up to the time of admission. The pain was "sharp and jabbing" in character, and did not radiate into the back or shoulders.
The bowels had moved normally on the morning of admission before the onset of the pain, but after this there was no further evacuation. Micturition was normal, and not in any way affected by the pain.

Most of the previous history was elicited a few days after the operation, for when seen after admission she was too ill to give any coherent story. She had suffered from " stomach trouble," which she described as biliousness, off and on for years, beginning at the age of about 25 . It took the form of mild attacks of epigastric pain coming on two to three hours after food, sometimes persisting until the next meal, the taking of which brought relief; at other times she had nausea, and was relieved by vomiting a small quantity of bilious material. The average duration of one of these attacks was a week, and she had several in a year. She had not observed any relation between these attacks and the ingestion of some particular food. Fatty focds she liked and they agreed with her. Her bowels had always been regular; there was no history of melaena nor had her stools ever been clay-coloured. She had had no trouble with micturition. Her menstrual function was free from any irregularities. Apart from her stomach trouble she had never had any illnesses or accidents in her life. She had never been jaundiced or even sallow, nor-so far as she knew -had she ever had typhoid fever or a pyrexia of unknown origin. She had one child, now 22 years of age, who had always been healthy. No miscarriages or abortions.

On examination she was in great pain, and showed some degree of shock. Pulse 120 beats per minute and of low tension; temperature $97.5^{\circ}$; hands cold; slight cold sweat on forehead ; tongue thinly coated with white fur.

The abdomen was somewhat scaphoid in appearance. Immobility of the abdominal wall was present, respiratory movement being entirely thoracic in character. There was a generalized, board-like rigidity, with tenderness in the epigastrium, most marked over the right rectus at a level about midway between the umbilicus and the xiphisternum. No tumour could be felt. On percussion there was no apparent loss or increase of liver dullness, while no dullness was present in either flank. The clinical picture seemed to be that of a clear-cut case of acute perforation of a peptic ulcer, and this diagnosis was accordingly made.

\section{Operation}

A mid-line supra-umbilical incision was made. On opening the peritoneal cavity there was no escape of gas. No ulcer was present either in the stomach or in the duodenum, and there was no evidence of peritonitis. It was noticed that the first and second parts of the duodenum and the pyloric part of the stomach were pushed forward, and the proximal half of the transverse colon displaced downwards. The agent responsible for this was a large globular cystic swelling about $12 \mathrm{~cm}$. in diameter. Above it the gall-bladder could be seen lying distended, and normal in size and appearance. The liver showed no sign of disease.

The cyst was covered with peritoneum continuous with the gastro-hepatic omentum and with that covering the duodenum. It extended above to the hilum of the liver, below to the lower end of the second part of the duodenum, to the left nearly as far as the mid-line, and to the right as far as the edge of the liver. The cyst was found to extend posteriorly to the right kidney, but a hand could easily be passed between it and the lower two-thirds of the anterior surface of the kidney. Anteriorly it was pushing the head of the pancreas forwards as well as the duodenum. The foramen of Winslow was not found, and the exact relation of the cyst to the cystic duct could not be defined. The cyst was thought at first to be pancreatic in origin, but on aspiration with a small needle cloudy bile was withdrawn, this making it clear that the condition was one of cystic dilatation of the common duct. Seeing that the bile looked infected, and that the condition of the patient was not good, it was decided to drain the cyst and leave any further operative procedure until a later date. A trocar and cannula was inserted into the anterior wall of the cyst above and to the right of the duodenum. On withdrawal of the trocar about eight ounces of fluid were evacuated. The cannula was then removed, and the hole made by it enlarged sufficiently to admit the index finger. Owing to the large size of the cyst the whole of its 
inner wall could not be palpated, but the portion felt was smooth. So far as one could judge, the wall of the cyst was of the same thickness as that of a normal common duct. No communications between the cyst and gall-bladder, hepatic duct, or duodenum could be found. A small tube was then inserted into the cyst through the enlarged trocar puncture wound, the edge of which was sutured tightly around the tube. The abdominal wall was then closed in layers, the tube being brought out through the inferior angle of the wound.

\section{Subsequent History}

The patient was far from well on the day following the operation, but later began to make excellent progress. After two days the bile lost its cloudy appearance and became clear and normal-looking. The faeces were normal in colour.

Dr. H. J. Slade, bacteriologist to the Infirmary, reported as follows on the specimen of bile sent him: lactosefermenting and non-lactose-fermenting bacilli were grown in the cultures from the bile. The lactose-fermenters were typical of $B$. coli communis. The characteristics of the non-lactosefermenters were as follows: motile with progression and Gramnegative. Sugar fermentations: as for $B$. typhosus. Agglutination: positive for $B$. typhosus. Conclusions: $B$. coli and $B$. typhosus infection. The Widal reaction was negative for $B$. paratyphosus, but strongly positive for $B$. typhosus in dilutions of 1 in 25,1 in 50 , and slightly so in a dilution of 1 in 125.

As a consequence of these findings the patient was removed on September 16th to the Newcastle-upon-Tyne City Hospital for Infectious Diseases, Walkergate, where she was under the care of Dr. Basil Dormer, who has kindly supplied me with the following details of her progress. On admission the tube was draining freely. The sutures were removed on September 19th, and the tube came out of the wound on September 21st (twelve days after operation). B. typhosus was again isolated from the bile; a specimen of faeces examined for this organism was negative. A Widal test showed agglutination in a dilution of 1 in 125. She appeared to make an uninterrupted recovery until about 2 p.m. on September 26th, when she complained of feeling unwell and of stiffness in the limbs. There was no pain or other localizing symptom in the chest or abdomen. On examination the pulse was imperceptible and the patient cyanosed. Digitalis and strychnine were injected subcutaneously and oxygen was administered, but the patient died at 5.15 p.m.

\section{Post-mortem Findings}

The wound had healed by first intention. A small hole with slightly reddened edges at the infsior angle indicated the drainage opening. The wound was reopened and slightly enlarged. The anterior wall of the cyst around its drainage opening was adherent to the anterior abdominal wall. The adhesions were separated, and a clear view of the abdominal cavity was obtained. There was no evidence of infection extravasation of bile, or other abnormality. The cyst was collapsed and, together with its connexions above and below, was removed en masse in order that its relations might be more carefully studied. Fig. 1 (on Plate) is a photograph of the specimen obtained, the cyst and duodenum having been cut open. The cyst had shrunk to about the size of a tangerine orange. In its upper part were seen two openings. The larger led into the common hepatic duct, which divided into its two branches about half an inch above its junction with the cyst. Above the cyst there was no dilatation of the extraor intra-hepatic bile ducts. The smaller opening led into the cystic duct, which was not dilated. It was seen to join the cyst independently of the common hepatic duct. At the lower end of the cyst was a small slit-like opening. A probe introduced into this passed obliquely downwards and to the right through the duodenal wall, and emerged into the lumen of the duodenum in the usual position. On cutting open this part of the duct it was found to be narrower than normal, but the ampulla was well defined, and the small opening of the pancreatic duct was seen just above it.

From the above description it will be seen that the cystic dilatation involved the whole of the extra-duodenal portion of the common duct, and also the lower end of the common hepatic duct. The small intestine was opened and found to contain bile-coloured faecal matter. The Peyer's patches showed no evidence of inflammation or ulceration. The heart was not dilated, and no ante-mortem thrombus or embolus was present. The myocardium was pale and flabby, and the coronary arteries were free from disease. The lungs showed no evidence of embolism or thrombosis, nor was pneumonia or congestion present. Permission to examine the brain was refused. The above findings are insufficient to establish the cause of death.

\section{Commentary}

Certain interesting features of this condition seem worthy of mention.

Females predominate in a proportion of nearly 5 to 1. The age at which the condition has been found varies considerably, one case having been reported in a stillborn foetus, while the oldest patient was 66. The majority of cases, however, have come to operation in late adolescent or early adult life.

As to the pathological anatomy, the cyst is formed from a dilatation of the extra-duodenal portion of the common duct, and also, as in the present case, of the lower portion of the common hepatic duct. The right and left hepatic ducts are not dilated, nor is the intramural portion of the common duct usually affected, although in some cases there has been a diminution, and in others an increase, in the diameter of this portion of the duct. The liver usually shows no pathological change, but in a few cases a mild degree of biliary cirrhosis has appeared to exist. This type of dilatation is therefore very different from that due to obstruction of the common duct by stones or disease of the pancreas. In this case the common duct is uniformly enlarged throughout its whole length above the site of obstruction, while the dilatation also involves the hepatic ducts, the diameter of which is increased more or less proportionately to that of the common duct. The common duct may dilate to the size of a loop of small intestine, but rarely, even in advanced cases, does it become larger. A cyst of the common duct, on the other hand, may assume enormous proportions. Although the smallest one reported was about 3 by $2 \frac{1}{2} \mathrm{~cm}$., in most instances the cyst is usually much larger, varying in size from a tangerine orange to a man's head. A capacity of four to five litres has been recorded. The duodenum is pushed forwards and medially by the cyst, which extends for a variable distance to the left behind the pancreas and pyloric part of the stomach. The hepatic flexure and right half of the transverse colon are displaced downwards.

As regards etiology the two principal hypotheses which have been advanced in explanation of the formation of a cyst of the common duct are, first, that of intermittent obstruction in the lower part of the duct with consequent dilatation above, and, secondly, that which regards the condition as a congenital anomaly. The first theory is based upon the fact that in some cases the direction of the intramural portion of the common duct differed from the normal, or else there was present a valve-like duplication of the wall of the cyst at its opening into this terminal part of the duct (Waller ${ }^{7}$ et alii). It is thought that when the cyst is filled to a certain degree either of these conditions produces a kink, leading to a temporary obstruction to the flow of bile into the duodenum. On further distension of the cyst, due to the continuous flow of bile into it from the liver, the kink is freed, and bile is thus allowed to pass from the cyst into the duodenum. While this mechanism offers an explanation of the intermittent jaundice observed in these cases, there seems no convincing evidence in favour of its being the causative factor in the production of the dilatation of the common duct. Indeed it would seem, for the following reasons, that the abnormalities noted are more likely to be a result of the dilatation than its cause. Intermittent obstruction in a hollow muscular system always leads to a uniform dilatation, above the obstruction, of the system affected. 
We know that the intermittent obstruction caused by a stone in the ureter leads to dilatation both of the ureter above and also of the pelvis and calyces of the kidney. To make a more pertinent observation, the dilatation caused by a stone in the common duct in the same way not only affects that part of the common duct above it, but extends upwards along the extra- or intra-hepatic bile ducts. However, in cases of common duct cyst the dilatation ceases abruptly in the region of the common hepatic duct and is not continued upwards, the hepatic ducts above the cyst being unaffected. Further, it would be difficult to give credence to intermittent obstruction as the cause of cyst formation in Fowler's case, ${ }^{8}$ in which the lumen of the intramural portion of the common duct was dilated to such an extent that the operator was able to pass his finger from the cyst into the duodenum. An additional objection to this hypothesis is to be found in the absence of jaundice in the present case, an indication that no obstruction to the flow of bile into the duodenum had ever existed-this, in spite of the fact that the lumen of the terminal portion of the common duct was narrower than usual.

It seems, on the whole, that the condition should be regarded as a congenital anomaly until further evidence may disprove this hypothesis. The fact that such a cyst has been found in a foetus (Heiliger ${ }^{9}$ ) supports this opinion. The condition appears to be analogous to the so-called idiopathic dilatations which have been found in other hollow muscular systems. The ultimate causative factor, whether primarily muscular or nervous in origin, still remains unknown.

\section{Symptomatology}

In most cases the onset of symptoms takes place in childhood, and from that time they appear intermittently. In a typical case the patient suffers from intermittent attacks consisting of the triad, upper abdominal pain, vomiting, and jaundice, and the presence of a palpable tumour in the right hypochondrium. The pain may vary in intensity from a mildness causing the patient little worry to a severity requiring morphine for its relief. It usually occurs in the right hypochondrium, but its radiation into the back and shoulder is variable. Vomiting nearly always accompanies the pain, and may be severe enough to cause great distress. Jaundice has proved to be the most constant symptom in this condition, and although it may be present only as a slight icteric tinge, yet in nearly all the recorded cases it has been of marked degree. 'The presence of a tumour, more especially if it appears while an attack is under observation, as in Taylor's case, ${ }^{5}$ can be regarded as probably the most significant diagnostic factor. Only in a few cases has it been recorded as absent. When present the tumour may vary considerably in size, sometimes being just palpable, at other times extending from the costal margin above to the crest of the ilium below. Up to the present time the condition has been diagnosed prior to operation in only three cases. The many diagnoses which have been made include hydatid cyst (the most frequent), pancreatic cyst, diseases of the gall-bladder and ducts other than the cystic, cyst or abscess of the liver, hydronephrosis, and ovarian cyst. It is now established that the intermittent appearance of the clinical triad mentioned above, together with the presence of a tumour in the right hypochondrium, occurring in a young individual, especially a female, should point to the diagnosis of cystic dilatation of the common bile duct.

\section{Unusual Features}

Having described briefly the clinical features typical of the condition, it will suffice to draw attention to the uncommon characteristics of the present case. (1) The patient was of middle age. (2) She was perfectly healthy throughout her childhood and adolescence, the gastric symptoms not beginning till the age of 25 . (3) Jaundice was never present at any time. (4) No tumour was palpable. (This, however, is not surprising, considering the board-like rigidity of the abdominal wall, and it is not possible to say whether or not a tumour would have been felt had the patient been examined when in ordinary health or during one of her bilious attacks.) (5) Clinically, the condition of the patient, on the first occasion on which she had had need of medical attention, resembled that of acute perforation of a peptic ulcer. The cause of the patient's illness was apparently an acute cholangitis. The bile contained $B$. typhosus and $B$. coli. The Widal reaction two days after operation showed an agglutination in a dilution up to 1 in 125 ; a week later the agglutination remained the same. This finding leads one to the conclusion that the patient was a typhoid carrier, and was not suffering from an acute typhoidal infection, for in such a case the Widal reaction after nine days would probably have shown agglutination in a dilution in at least 1 in 1,000 . With $B$. typhosus as the infective agent thus disposed of, it would appear that the causative organism of the acute cholangitis was the B. coli.

\section{TREATMENT}

There is no doubt that the treatment of choice is the performance of an anastomosis through which bile may pass easily into the upper gastro-intestinal tract and so reduce stasis and lessen the tendency to cholangitis. The results in cases treated by this method of primary anastomosis have been much more successful than those in which other types of treatment have been employed. The anastomosis most commonly advocated is a choledochoduodenostomy, but a cholecysto-gastrostomy appears to be equally efficacious. Other operative procedures, such as aspiration (which obviously can be only a temporary measure) and marsupialization of the cyst, have not provèd satisfactory. External drainage of the cyst as the sole procedure has not met with much success, death resulting in some cases from peritonitis or haemorrhage, while in those patients who have lived a biliary fistula has sometimes persisted for months, only for its eventual closure to be followed by a recurrence of symptoms. In several instances, however, the performance of secondary anastomosis between the cyst and the duodenum or jejunum following a preliminary drainage has proved satisfactory. In the present case it was intended that a choledocho-duodenostomy should be performed at an early date after the patient had recovered fully from the effects of her acute illness. Everything seemed propitious for the carrying out of that intention, when the patient died suddenly from causes apparently unassociated with the condition for which she had been operated upon.

I wish to thank Mr. J. Hamilton Barclay, into whose clinic the patient was admitted, for his permission to publish this case.

\section{REFERENCES}

1 McConnell, A. A.: Cyst of the Common Bile Duct, Brit. Journ. of Surg., 1919-20, vii, 520-524.

2 Judd, E. S., and Greene, F. I.: Choledochus Cyst (Bibliography), Surg., Gynecol. and Obstct., 1928, xlvi, 317-324.

3 Wilson, H. V. P., jun.: Choledochus Cyst, Journ. Amer. Med. Assoc., 1930, xcv, 399-400.

4 Neugebauer, F.: Zur Kenntnis der Idiopathischen Choledochuscyste, Beitr. $z$. klin. Chir., 1924, cxxxi, 448-455.

5 Taylor, J.: Cystic Dilatation of the Common Bile Duct, Brit. Journ. of Surg., 1928-29, xvi, 327-332.

'Iselin, H.: Gemeinsame Cyste der Gallen und Pankrcaswege, Arch. f. klin. Chir., 1S27, cxlv, 304-309.

-Waller, E.: Idiopathic Cholcdochus Cyst, Annals of Surgery, 1917, lxvi, 446-463.

${ }^{8}$ Fowler, R. S.: Choledochus Cyst, Annals of Surgery, 1916, lxiv, $546-549$

๑ Heiliger: Quoted by Waller. 
FEbroary 6, 1932]

JAMES H. SAINT : CYSTIC DILATATION OF COMMON BILE-DUCT

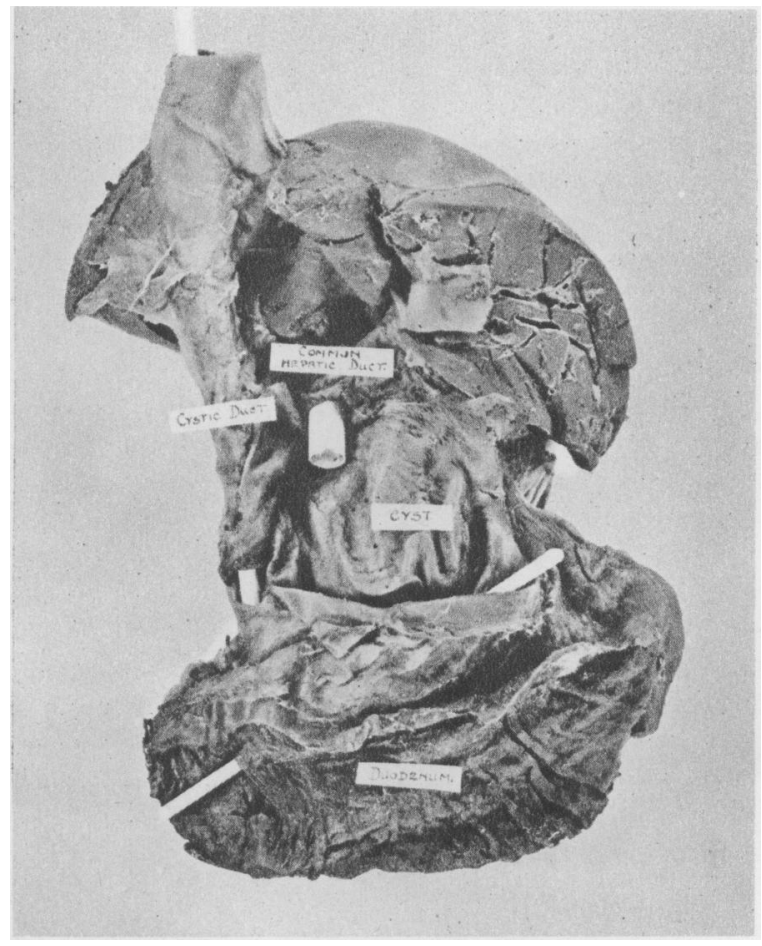

Fra. 1.-Specimen showing the opened dilated common duct and its relation to surrounding structures.
ANDREW FOWLER: ADENOMATOUS PAPILLOMA OF THE DUODENUM

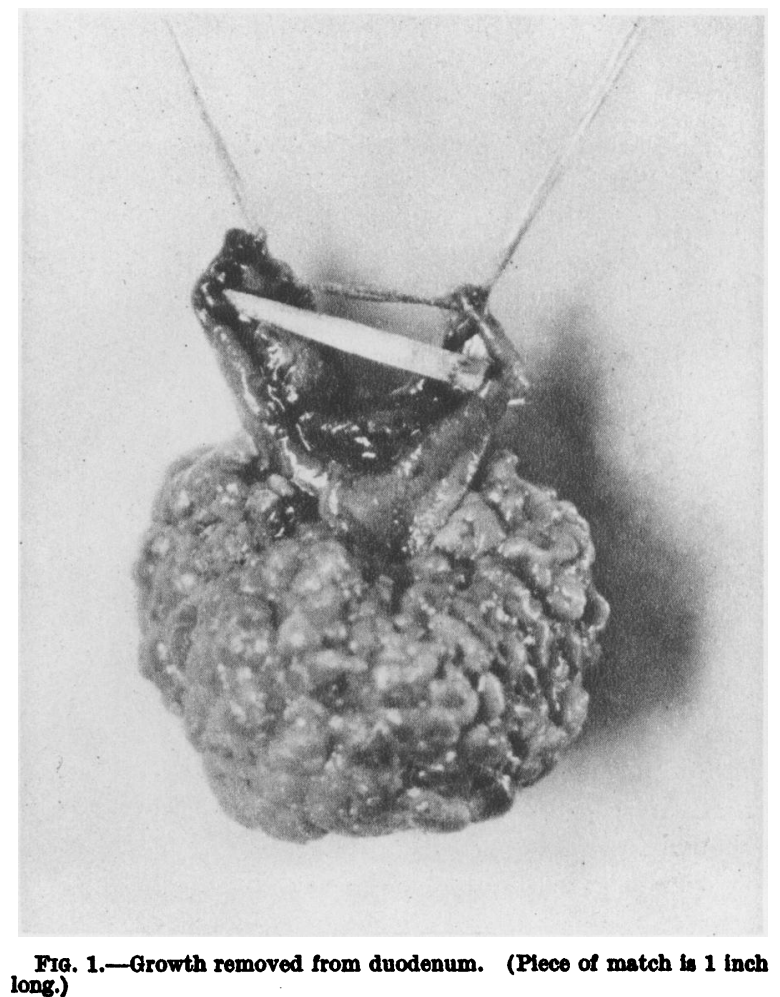

ANDREW FOWLER : ADENOMATOUS PAPILLOMA OF THE DUODENUM

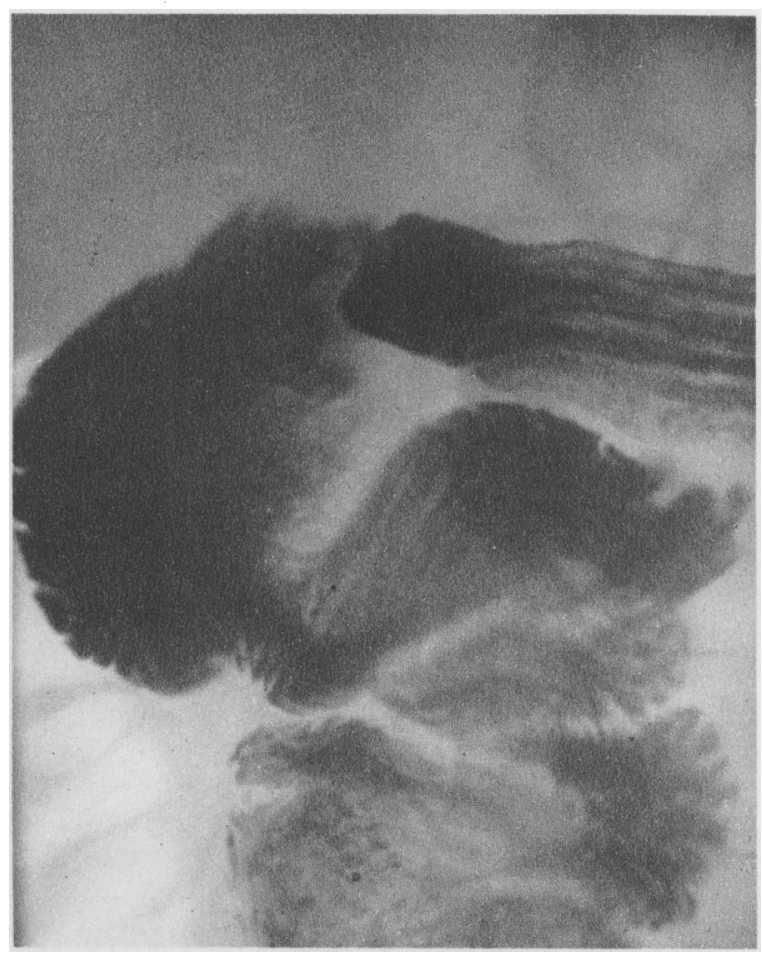

Fic. 2.-First radiograph, showing contracted cap with dilatation of dewcending limb of duodenum.

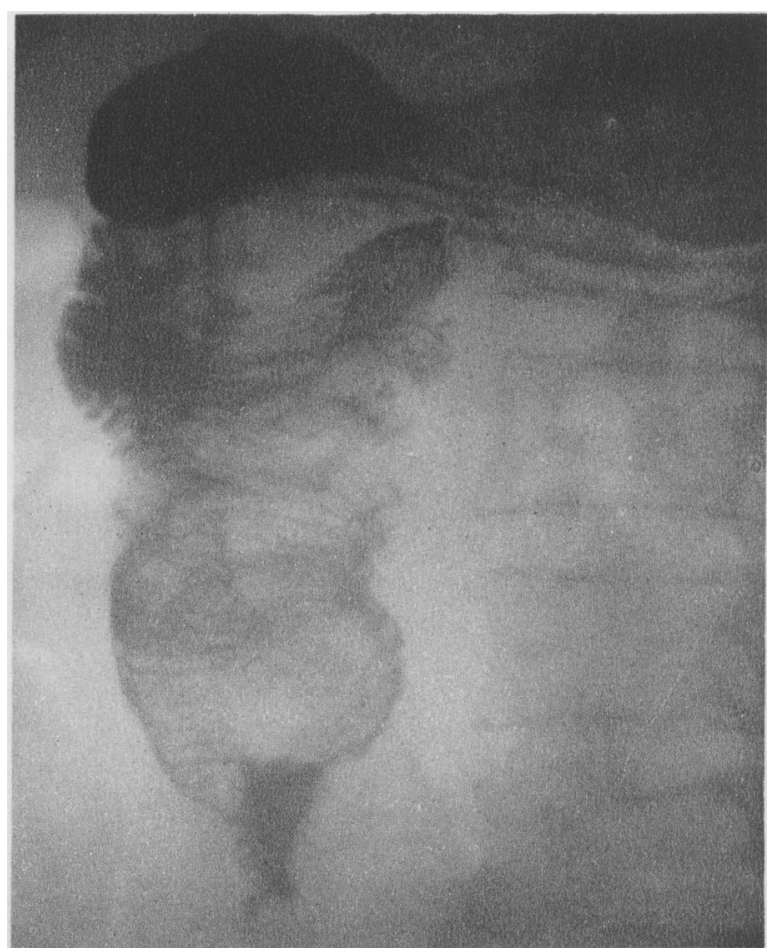

FTG. 8. - Second radlograph (10 minutes later), thowing dilatation of jofunum with constriction below. [Relatively reduced.] 